\title{
Gravitational Collapse of Massless Fields in an Expanding Uni- verse
}

\author{
Chul-Moon Yoo ${ }^{1, \star}$ \\ ${ }^{1}$ Division of Particle and Astrophysical Science, Graduate School of Science, Nagoya University, Nagoya \\ 464-8602, Japan
}

\begin{abstract}
Gravitational collapse of a massless scalar field with the periodic boundary condition in a cubic box is reported. This system can be regarded as a lattice universe model. The initial data is constructed for a Gaussian like profile of the scalar field taking the integrability condition associated with the periodic boundary condition into account. For a large initial amplitude, a black hole is formed after a certain period of time. While the scalar field spreads out in the whole region for a small initial amplitude. The difference of the late time expansion law of the lattice universe depending on the final fate of the gravitational collapse is discussed.
\end{abstract}

\section{Introduction}

When we consider gravitational collapse, the most common boundary condition is the asymptotically flat boundary condition. Obviously, the asymptotically flat boundary condition is relevant for stellar collapse. Nonetheless, when we consider collapse of a cosmological object, we may not neglect the effect of the expansion of the universe. For instance, for simulation of a primordial black hole, whose horizon scale may be comparable to the Hubble horizon scale. Here, we report a numerical experiment of gravitational collapse in an expanding background.

In this paper, we consider a massless scalar field in a cubic box with the periodic boundary condition for each pair of opposite boundary surfaces. This system can be regarded as a lattice universe model in which the same cubic region is spatially repeated. Black hole systems with the same boundary condition is studied in Refs. [1-3]. In practice, we simulate the only octant region of the cubic box imposing reflecting boundary condition on each boundary surface. The initial scalar field configuration is set to be a Gaussian like profile. Then, we report how the dynamics of the system changes depending on the initial amplitude of the scalar field profile focusing on black hole formation and expansion law of the lattice universe.

In Sec. 2, settings and initial data construction are given. In Sec. 3, we report the time evolution for large amplitude and small amplitude cases. We use the geometrized units in which both the speed of light and Newton's gravitational constant are one.

\footnotetext{
^e-mail: yoo@gravity.phys.nagoya-u.ac.jp
} 


\section{Settings and initial data construction}

\section{1 scalar field}

We consider the massless scalar field, whose equations of motion are given by $\nabla^{\mu} \nabla_{\mu} \phi=0$. Introducing the momentum $\Pi:=-n^{\mu} \nabla_{\mu} \phi$, we can rewrite the field equations in the form of the $3+1$ decomposition as follows:

$$
\begin{aligned}
& \left(\partial_{t}-\beta^{i} \partial_{i}\right) \phi=-\alpha \Pi, \\
& \left(\partial_{t}-\beta^{i} \partial_{i}\right) \Pi=-\alpha \Delta \phi-\gamma^{\mu v} \partial_{\mu} \alpha \partial_{\nu} \phi+\alpha K \Pi,
\end{aligned}
$$

where $\alpha, \beta^{i}, \gamma^{\mu \nu}$ and $K$ are the lapse function, shift vector, spatial metric and the trace of the extrinsic curvature $K_{i j}$.

The stress energy tensor $T_{\mu \nu}=\nabla_{\mu} \phi \nabla_{\nu} \phi-g_{\mu \nu}\left(\frac{1}{2} \nabla^{\rho} \phi \nabla_{\rho} \phi\right)$ can be also expressed in the form of the $3+1$ decomposition as follows:

$$
\begin{aligned}
\rho & :=n_{\mu} n_{\nu} T^{\mu \nu}=\frac{1}{2} \Pi^{2}+\frac{1}{2} \mathrm{e}^{-4 \psi} \tilde{\gamma}^{i j} \partial_{i} \phi \partial_{j} \phi, \\
j_{i} & :=\quad-\gamma_{i \mu} n_{\nu} T^{\mu \nu}=-\Pi \partial_{i} \phi, \\
S_{i j} & :=\gamma_{i \mu} \gamma_{j v} T^{\mu v}=\partial_{i} \phi \partial_{j} \phi-\frac{1}{2} \tilde{\gamma}_{i j} \tilde{\gamma}^{k l} \partial_{k} \phi \partial_{l} \phi+\frac{1}{2} \mathrm{e}^{4 \psi} \tilde{\gamma}_{i j} \Pi^{2},
\end{aligned}
$$

where $\gamma_{i j}=\mathrm{e}^{4 \psi} \tilde{\gamma}_{i j}$.

\subsection{Initial data}

Constraint equations are given by the followings:

$$
\begin{array}{cl}
\mathcal{R}+K^{2}-K_{i j} K^{i j} & =16 \pi \rho, \\
D_{j} K^{j i}-D^{i} K & =8 \pi j_{i} .
\end{array}
$$

We adopt the conventional decomposition given by $K_{i j}=\mathrm{e}^{4 \psi} \tilde{A}_{i j}+\frac{1}{3} K \gamma_{i j}$. The momentum constraints are trivially satisfied under the following assumptions: $\Pi=0, K=$ const., $\tilde{\gamma}_{i j}=\delta_{i j}, \tilde{A}_{i j}=0$. The Hamiltonian constraint is given by

$$
\Delta \psi+\delta^{i j} \partial_{i} \psi \partial_{j} \psi-\frac{1}{12} K^{2} \mathrm{e}^{4 \psi}=-2 \pi \rho \mathrm{e}^{4 \psi}=-\pi \delta^{i j} \partial_{i} \phi \partial_{j} \phi
$$

We consider the following Gaussian like initial scalar field profile:

$$
\phi_{0}=A \exp \left[-\frac{r^{2}}{2 \sigma^{2}}\right] W(r)
$$

where $L$ is the length of the edge of the cubic box and $W(r)$ is a function to suppress the Gaussian tail and make it compatible to the periodic boundary condition.

In order to solve the equation (8), we need to impose the following integrability condition:

$$
0=\int_{\text {box }} \mathrm{d}^{3} x\left(\Delta \psi-\frac{1}{12} K^{2} \mathrm{e}^{4 \psi}+(\partial \psi)^{2}+\pi(\partial \phi)^{2}\right) \Leftrightarrow K^{2}=12 \frac{\int_{\text {box }} \mathrm{d}^{3} x\left((\partial \psi)^{2}+\pi(\partial \phi)^{2}\right)}{\int_{\text {box }} \mathrm{d}^{3} x \mathrm{e}^{4 \psi}},
$$


where the integral of the Laplacian part vanishes because of the periodic boundary condition. We numerically solve the elliptic differential equation (8) by using iterative method imposing the integrability condition at each step of the iteration. The length scale of the coordinates are fixed by imposing $\psi(L, L, L)=0$. We numerically solve the Hamiltonian constraint equation imposing the integrability condition.

We set $\sigma=0.3 L$ and consider the following two cases:

- Case $1: A \simeq 2.35$,

- Case2: $A \simeq 0.235$.

The initial profile of $\phi$ and $\psi$ are shown in Figs. 1 and 2 for the case 1 and 2, respectively. The initial
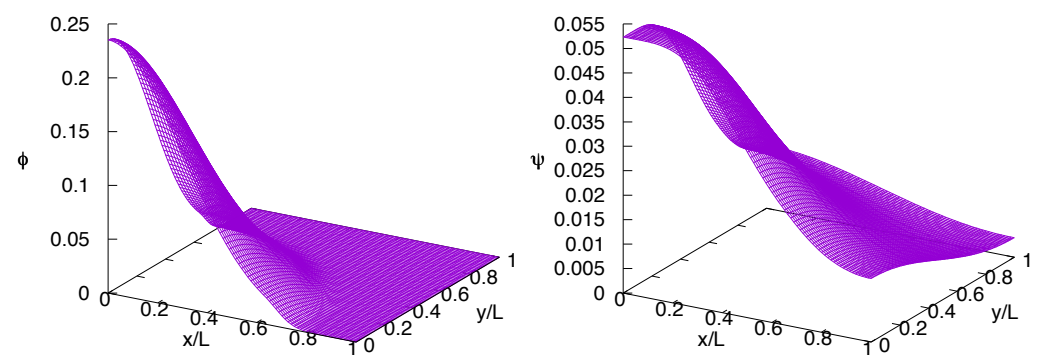

Figure 1. Initial profiles of $\phi$ and $\psi$ for the case 1.
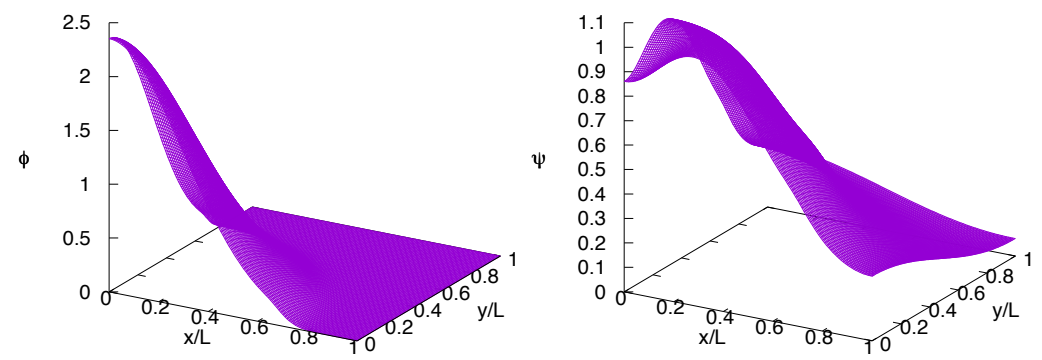

Figure 2. Initial profiles of $\phi$ and $\psi$ for the case 2 .

values of $1 / H:=3 /|K|$ are given by $3.62 L$ for the case 1 and $0.843 L$ for the case2.

\section{Time evolution}

For the simulation of the time evolution, we use the 4th order Runge-Kutta method with the so-called BSSN formalism $[4,5]$. We use the same gauge condition as in Ref. [2]. The grid spacing is uni-grid with Cartesian coordinates. Excision of a black hole interior region[6] is performed when a black hole is formed. 

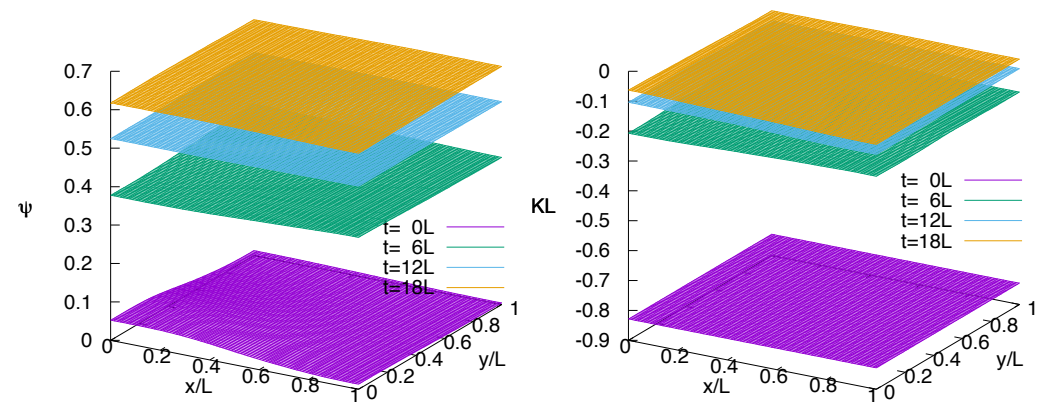

Figure 3. Evolution of $\psi($ left $)$ and $K($ right) for the case 1 .

\subsection{Case 1:small initial amplitude}

We show the time evolution of $\psi$ and $K$ on the $x-y$ plane in Fig. 3. It can be seen that the value of $\psi$ increases with time and the value of the effective Hubble parameter $-K / 3$ decreases with time. These behaviors explicitly show the expansion of the lattice universe.

\subsection{Case 2:large initial amplitude}

For the case 2, we observe black hole formation. Here, we show the time evolution of the shape of the horizon in the coordinate space in Fig. 4. The horizon is shrinking in the coordinate space as is also reported in Refs. [2, 3].

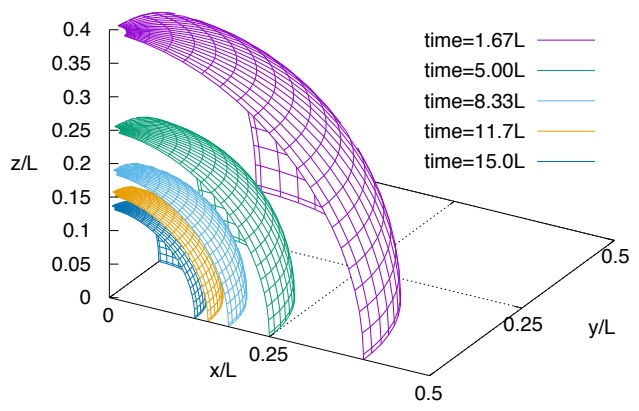

Figure 4. Evolution of the horizon shape in the coordinate space.

\subsection{Time evolution of the effective scale factor}

Finally, we show the dependence of the expansion law on the initial amplitude of the scalar field profile. We consider a set of observers each of which stays at a point of fixed spatial coordinates. Then, we calculate the area of a boundary surface on the constant proper time slice for the set of observers. Fig. 5 shows the time dependence of the square root of the area of a boundary surface. For the case 1 , the dependence is approximated by the radiation dominated universe $\left(\propto \tau^{1 / 2}\right)$ in a late 
time. While, for the case 2, the numerical result looks approaching that of the matter dominated universe $\left(\propto \tau^{2 / 3}\right)$.
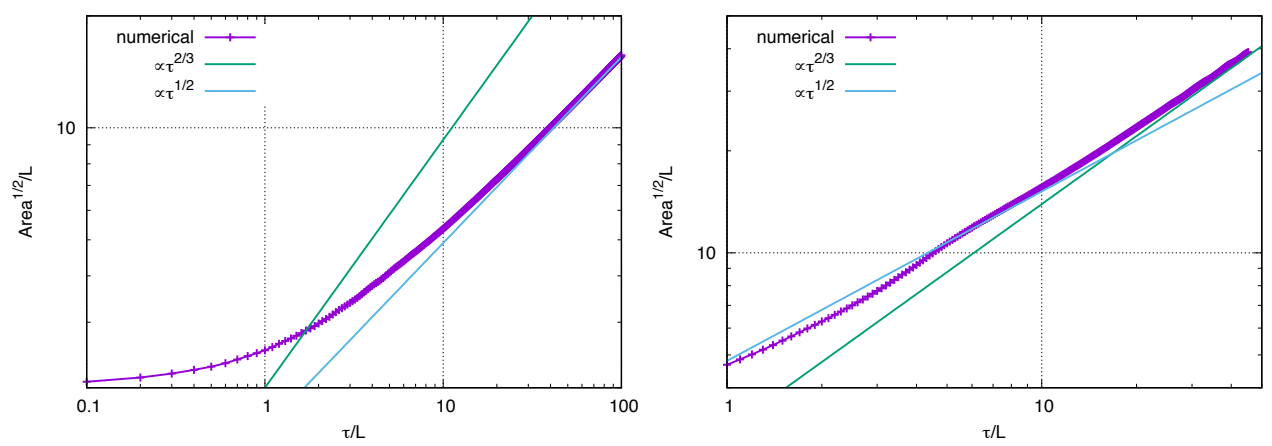

Figure 5. Square root of the area of a boundary surface is depicted as a function of the proper time measured by a set of observers with fixed spatial coordinates.

\section{4 summary}

We have considered gravitational collapse of a scalar field with the periodic boundary condition in a cubic box. This system can be regarded as a lattice universe model. We have shown that, when the initial amplitude of the scalar field is so small that the scalar field can finally spread in the whole region, the expansion law of the lattice universe model can be approximated by that of the radiation dominated universe in a late time. While, when the initial amplitude is so large that a black hole can be formed, the expansion law approaches that of the matter dominated universe. The all results are preliminary and we will report more concrete results elsewhere.

\section{Acknowledgements}

This work was supported by JSPS KAKENHI Grant Numbers JP16K17688, JP16H01097 (CY),

\section{References}

[1] C.M. Yoo, H. Abe, K.i. Nakao, Y. Takamori, Phys.Rev. D86, 044027 (2012), 1204 . 2411

[2] C.M. Yoo, H. Okawa, K.i. Nakao, Phys.Rev.Lett. 111, 161102 (2013), 1306.1389

[3] C.M. Yoo, H. Okawa, Phys. Rev. D89, 123502 (2014), 1404. 1435

[4] M. Shibata, T. Nakamura, Phys.Rev. D52, 5428 (1995)

[5] T.W. Baumgarte, S.L. Shapiro, Phys.Rev. D59, 024007 (1999), gr-qc/9810065

[6] M. Alcubierre, B. Bruegmann, Phys. Rev. D63, 104006 (2001), gr-qc/0008067 